LUMBAR PUNCTURE IN THE NEONATE

The diagnostic utility of lumbar punctures in neonates was evaluated in the Division of Neonatology, Department of Pediatrics, University of Miami, FL. Of 712 neonates with lumbar punctures during the first week of 1 ife, 8 had positive spinal fluid cultures but only 1 had a positive blood culture and a clinical course consistent with meningitis. In contrast, of 114 patients who underwent lumbar punctures after the first week of 1 ife, $7 \%$ had a positive culture approximating 5 times that observed during the first week of life. (Schwersenski $J$ et al. Lumbar puncture frequency and cerebrospinal fluid analysis in the neonate. AJDC Jan 1991; $145: 54-58$ ).

COMMENT. The authors conclude that the routine use of lumbar puncture as a part of the work-up for infection during the first week of life in an asymptomatic neonate should be reassessed. The results of the study suggest that lumbar puncture may not be justified in an asymptomatic at-risk neonate during the first week of life but after the first week lumbar puncture may be indicated in the at-risk infant.

\title{
NEUROLOGIC COMPLICATIONS OF CAT-SCRATCH DISEASE
}

The neurologic complications of cat-scratch disease in 76 patients with an average age of 10 years are reported from the Jacksonville Health Education Program, University of Florida, and Department of Pediatrics, Uni formed Services, Bethesda, MD. The incidence of encephalopathy varied from $0.17 \%-2 \frac{\circ}{\circ}$ in two groups of patients examined. Convulsions occurred in 28 (46\%) of 61 patients, and combative behavior occurred in $39 \%$ of those who were semi-conscious during the recovery phase of a convulsive episode. Headache, lethargy and malaise were cammon and persistent. Less cammon findings were ataxia, bilateral VI nerve palsy, aphasia, transient hemiplegia, and profound hearing loss lasting eight months. Of 15 patients without encephalopathy two had facial nerve paresis, ten neuroretinitis, and three peripheral neuritis. All 76 patients recovered within 12 months and $78 \%$ recovered within 1-12 weeks. There were no neurologic sequelae. Treatment with antibiotics and prednisone is usually ineffective. The authors recommend IV Gentamycin in severely ill patients. (Carithers HA, Margileth AM. Cat-scratch disease. Acute encephalopathy and other neurologic manifestations. AJDC Jan $1991 ; 145: 98-101)$.

COMMENT. Cat-scratch disease encephalopathy should be considered in a young patient presenting with sudden onset of coma and/or convulsions. The patient should be examined for lymphadenopathy and inoculation papule or conjunctival granulama. The diagnosis can be confirmed by a cat-scratch skin test. The differential diagnosis includes Lyme encephalitis, drug intoxication, viral or bacterial infections, and Reye's syndrome. 